\section{Cobertura do exame físico de mama: estudo de base populacional em Pelotas, RS}

\section{Coverage of physical breast examination to prevent breast cancer: a population-based study in Pelotas, RS}

\author{
Juvenal Soares Dias da Costa \\ Professor Adjunto \\ Departamento de Medicina Social \\ Faculdade de Medicina \\ Universidade Federal de Pelotas \\ Centro de Ciências da Saúde \\ Universidade do Vale do Rio dos Sinos \\ Av. Duque de Caxias, 250 CEP 96030-002 - Pelotas - RS \\ jcosta@epidemio-ufpel.org.br \\ Maria Teresa Anselmo Olinto \\ Centro de Ciências da Saúde \\ Universidade do Vale do Rio dos Sinos - UNISINOS

\section{Denise Petrucci Gigante} \\ Faculdade de Nutrição \\ Universidade Federal de Pelotas
}

\section{Ana Maria Baptista Menezes}

Departamento de Clínica Médica

Faculdade de Medicina

Universidade Federal de Pelotas

\section{Silvia Macedo}

Departamento de Clínica Médica

Faculdade de Medicina

Universidade Federal de Pelotas

\section{Sandra Costa Fuchs}

Departamento de Medicina Social

Faculdade de Medicina

Universidade Federal do Rio Grande do Sul

\section{Resumo}

Objetivos: Verificou-se a cobertura do exame físico de mama realizado por médicos, no último ano, nas mulheres com idade entre 20 e 69 anos, residentes na zona urbana da cidade de Pelotas. Identificaram-se características associadas à realização do procedimento. Os resultados foram comparados com outro estudo transversal de base populacional realizado em 1992.

Materiais e métodos: Realizou-se um estudo transversal de base populacional, utilizando-se amostragem por conglomerados. A regressão logística foi realizada seguindo modelo hierarquizado.

Resultados: Entre as 1122 mulheres de 20 a 69 anos incluídas no estudo, verificou-se que 549 (48,9\%) tiveram exame de mama realizado por médicos. Encontrou-se associação para classe social, cor da pele, freqüência de consultas médicas e realização de exame citopatológico.

Discussão: Abordou-se a falta de integralidade no sistema local de saúde. Discutiu-se as evidências da iniqüidade da atenção.

Palavras-chave: Exame físico. Mama. Epidemiologia. Estudos transversais. Avaliação. Avaliação de ações de saúde pública (processo e resultado). Avaliação de processos e resultados (cuidados de saúde). Avaliação de programas. 
Abstract

Objectives: The study aimed to evaluate breast exams performed by doctors, in the previous year, in women with ages ranging from 20 to 69 years. Some characteristics of this procedure were investigated. The results were compared with another populationbased cross-sectional study done in 1992.

Methods: A population-based cross sectional survey was conducted in an urban area of southern Brazil (Pelotas). The urban area of the city is comprised of 288 census tracts. A total of 40 census tracts were randomly selected for inclusion in the study. Logistic regression was performed following a hierarchical model of analyses.

Results: Among the 1,122 women included in the sample, 549 (48.9\%) had their breast exam performed by a doctor. An association was found for socioeconomic level, skin color, ambulatory attendance and cytopathologic tests.

Discussion: The dichotomy in the local health system and the evidences of inequalities in care were discussed.

Key Words: Physical examination. Self-examination. Breast. Epidemiology. Cross-sectional studies. Evaluation. Evaluation of public health interventions (process and outcome). Outcome and process assessment (health care).

\section{Introdução}

O câncer de mama é a principal neoplasia que acomete o sexo feminino no Brasil. A taxa bruta de incidência foi de 39,6 por 100.000 mulheres em 1999, enquanto a taxa de mortalidade foi de 9,2/100.000 ${ }^{1}$. Entre 1970 e 1995, a análise da série histórica de mortalidade por câncer em mulheres, no Rio Grande do Sul, mostrou que o câncer de mama apresentou o maior coeficiente. A mortalidade comportou-se de forma ascendente, sendo que o coeficiente de 7,1 por 100.000 habitantes, em 1970, elevou-se para 15,9 por 100.000 habitantes, em $1995^{2}$, até alcançar 16,5 no ano de $2000^{3}$.

Contudo, o diagnóstico precoce eleva a taxa de cura e aumenta a sobrevida das pacientes com câncer de mama ${ }^{4}$. Desta forma, deve-se empregar esforços na utilização de todos os métodos para o rastreamento de casos de câncer de mama. O exame físico de mamas pode ser realizado anualmente nas mulheres com idade entre 49 e 70 anos, ainda que não se encontrem evidências suficientes para contestar ou recomendar sua prática $^{5,6}$. Assim, o Programa de Controle do Câncer de Mama do Estado do Rio Grande do Sul preconiza o exame físico de mama para mulheres a partir dos $20 \operatorname{anos}^{7}$. O procedimento também tem sido utilizado como marcador de performance de serviços de saúde nos países desenvolvidos ${ }^{8,9}$.

A avaliação das políticas e ações desenvolvidas em saúde pública pode ser realizada através de estudos transversais que podem fornecer os níveis de cobertura dos programas ou atividades. Assim, pode-se implementar o processo de avaliação no início, antes da intervenção, para se saber o que deve ser mudado e, no final, para caracterizar se as ações foram efetivas ${ }^{10}$.

Assim, realizou-se um estudo com o objetivo de verificar a cobertura do exame médico de mama, relacionando-o com variáveis socioeconômicas, demográficas e de utilização de serviços de saúde. Os resultados deste estudo foram comparados com os obtidos em outro estudo transversal de base populacional realizado em $1992^{11}$. Fo- 
ram exploradas na análise as características das mulheres não examinadas, com o intuito de alertar o sistema local de saúde.

\section{Materiais e métodos}

Foi realizado um estudo transversal, de base populacional, envolvendo a investigação de diversos aspectos relacionados à saúde da população adulta, com idade entre 20 e 69 anos, residente na zona urbana da cidade de Pelotas, RS. O Projeto de Pesquisa foi aprovado pelo Comitê de Ética e Pesquisa da Faculdade de Medicina da Universidade Federal de Pelotas.

O tamanho da amostra foi estimado através de prevalências que variaram entre $25 \%$ e $75 \%$, através das quais pretendia-se investigar diversos desfechos. Assim, para cálculo do tamanho da amostra foi utilizado um poder de $80 \%$, um erro alfa de $5 \%$, para exposições variando entre $25 \%$ e $75 \%$, e com uma razão de prevalência de 1,6 que estimasse a magnitude do desfecho na população exposta. Foram acrescentados ao tamanho da amostra $10 \%$ pelas possibilidades de recusas e $15 \%$ para controle de fatores de confusão. Portanto, foi estimada uma amostra de 1.800 indivíduos. A seleção dos participantes foi feita através de visitas domiciliares em 40 setores censitários sorteados aleatoriamente. Em cada setor foram visitados 30 domicílios, totalizando 1.200 famílias, onde se esperava encontrar 1,5 pessoas na faixa etária do estudo. A partir de um quarteirão previamente sorteado, foi escolhido, também de maneira aleatória, o ponto de partida. Após a definição de um determinado domicílio, sistematicamente, saltava-se duas casas para então escolher a próxima.

Ao final do trabalho de campo foram encontradas $1.145(95,4 \%)$ das 1.200 famílias previstas. Destas, 57 (4,7\%) famílias incluíam indivíduos fora da faixa etária prevista no estudo, que foram excluídas da investigação. Além disso, outras 55 (4,5\%) famílias foram classificadas como perdas ou recusas. Foram encontradas 2.177 pessoas de 20 a 69 anos nos setores visitados, mas foram incluídos no estudo 1.968 participantes, de forma que as perdas e recusas individuais atingiram $9,6 \%$.

Do total da amostra foram identificadas $1.122(57,0 \%)$ mulheres entre 20 e 69 anos, que foram incluídas no presente estudo.

O trabalho de campo foi realizado entre dezembro de 1999 e abril de 2000, utilizando-se um questionário padronizado e précodificado, aplicado a todas as mulheres incluídas na pesquisa. Os entrevistadores eram acadêmicos da Universidade Federal de Pelotas, que foram treinados e avaliados durante estudo piloto. Os entrevistadores, desconhecendo os objetivos do estudo, coletaram informações sobre a realização de exame de mama por médico durante o último ano. Além destas informações, foram investigados: idade das mulheres, cor da pele, classe social, renda familiar per capita (em salários mínimos), escolaridade (em anos de estudo), número de consultas médicas durante o ano e local de consulta médica no último mês. Foram investigadas outras variáveis relacionadas com a utilização de serviços de saúde, tais como, internação hospitalar no último ano, realização do exame citopatológico nos últimos três anos e presença de hipertensão arterial sistêmica. Estas variáveis foram incluídas porque estudos epidemiológicos realizados na cidade de Pelotas revelaram que estas condições são marcadoras de uma maior utilização de serviços de saúde ${ }^{12,13}$. As variáveis foram coletadas com o mesmo enunciado da investigação realizada em 1992, o que garantiu a comparabilidade dos resultados ${ }^{11}$. Foram utilizadas duas classificações de classe social: a de Bronfman ${ }^{14} \mathrm{e}$ a da Associação Brasileira de Institutos de Pesquisa de Mercados $\left(\right.$ ABIPEME) ${ }^{15}$. A classificação da ABIPEME baseia-se na acumulação de bens materiais e escolaridade, e enquadra as pessoas nas classes A, B, C, D e E, a partir dos escores alcançados. A classificação de Bronfman categoriza os indivíduos a partir da propriedade dos meios de produção, de suas inserções nas atividades produtivas e de sua escolaridade.

A presença de hipertensão arterial sistêmica foi definida quando o entrevistado 
apresentava pressão arterial ${ }^{3} 160 / 95 \mathrm{~mm}$ Hg, obtida através da média de duas medidas, tomadas na metade e no final da entrevista, ou referia uso de medicação antihipertensiva. Os entrevistadores foram treinados em técnicas de aferição da pressão arterial, de acordo com o Consenso Brasileiro ${ }^{16}$. Foi realizada a medida de tensão arterial com esfigmanômetros aneróides, calibrados através de tensiômetro de mercúrio.

A variável freqüência de consultas no último ano foi analisada de forma dicotomizada (três ou mais consultas; uma a duas consultas), sendo excluídas da análise as 221 $(19,7 \%)$ mulheres que não haviam consultado no último ano.

A variável local de consulta foi coletada entre os diversos serviços possíveis e, posteriormente, agregada quanto à modalidade do financiamento e à intenção de lucro. A partir deste critério, foram formadas 4 categorias:

- sistema público: integrado pelos postos de saúde do bairro do indivíduo entrevistado, outros postos de saúde, ambulatório da Faculdade de Medicina e pronto-socorro;

- serviços contratados: constituídos pelos ambulatórios de hospitais com atendimento gratuito pelo Sistema Único de Saúde;

- seguros privados: formados pelos seguros privados de saúde, medicina de grupo e ambulatórios de sindicatos e empresas onde uma taxa era cobrada;

- sistema privado: constituído, exclusivamente, pelos médicos particulares.

Foi realizado controle para assegurar a qualidade das informações, aplicando-se um segundo questionário, simplificado, em 10\% das pessoas incluídas no estudo.

Após a codificação, foi realizada a dupla entrada de dados em um banco de dados do Programa Epi-Info, com o intuito de diminuir os erros de digitação. A análise dos dados foi realizada no Programa SPSS, onde foram comparadas as proporções de não realização do exame de mama por médico em relação às variáveis socioeconômicas, demográficas e de utilização de serviços de saúde. Foram calculadas as razões de prevalências e seus intervalos de confiança de 95\%, e foi testada a significância das associações através do teste do Qui-quadrado ${ }^{17}$. Foi ainda realizada regressão logística. seguindo-se modelo hierarquizado (Figura 1), para controle das variáveis de confusão ${ }^{18}$, calculando-se os odds ratio e seus intervalos de confiança.

\section{Resultados}

Entre as 1122 mulheres, com idade entre 20 e 69 anos, incluídas no estudo realizado no ano 2000, foi verificado que 549 (48,9\%; $\mathrm{IC}_{95 \%} 46,0$ a 51,8) não realizaram exame médico de mamas. Entretanto, no estudo realizado em 1992, foi constatado que entre as 934 mulheres entrevistadas, 149 (20,7\%; IC $_{95 \%}$ 18,1 a 23,3) não foram examinadas.

Conforme a descrição da amostra, foi verificada uma maior proporção de mulheres pertencente a classe $\mathrm{C}$, ao proletariado não típico, com renda mensal de um a três salário mínimos e com escolaridade até 4 anos (Tabela 1). Entre a população incluída no estudo, aproximadamente $83 \%$ das mulheres entrevistadas eram de cor branca, $69 \%$ tinham idade entre 35 e 69 anos e cerca de $50 \%$ consultaram três ou mais vezes durante $o$ ano.

Foi observada na análise univariada, apresentada na Tabela 1, a associação inversa entre classe social da ABIPEME e ausência de exame de mamas realizado por médicos. Dessa forma, à medida que diminuía a inserção de classe, aumentava o percentual de não realização do procedimento. A razão de prevalência da classe $\mathrm{E}$ foi quase três vezes maior que aquela encontrada na classe A, escolhida como categoria de base.

A associação entre classe social, segundo Bronfman, e a ausência de exame mostrou que, em comparação com a burguesia e a nova e pequena burguesia, as mulheres das demais classes apresentaram maior probabilidade de não serem examinadas por médicos (Tabela 1).

Quanto à renda familiar per capita, as diferenças foram estatisticamente significa- 


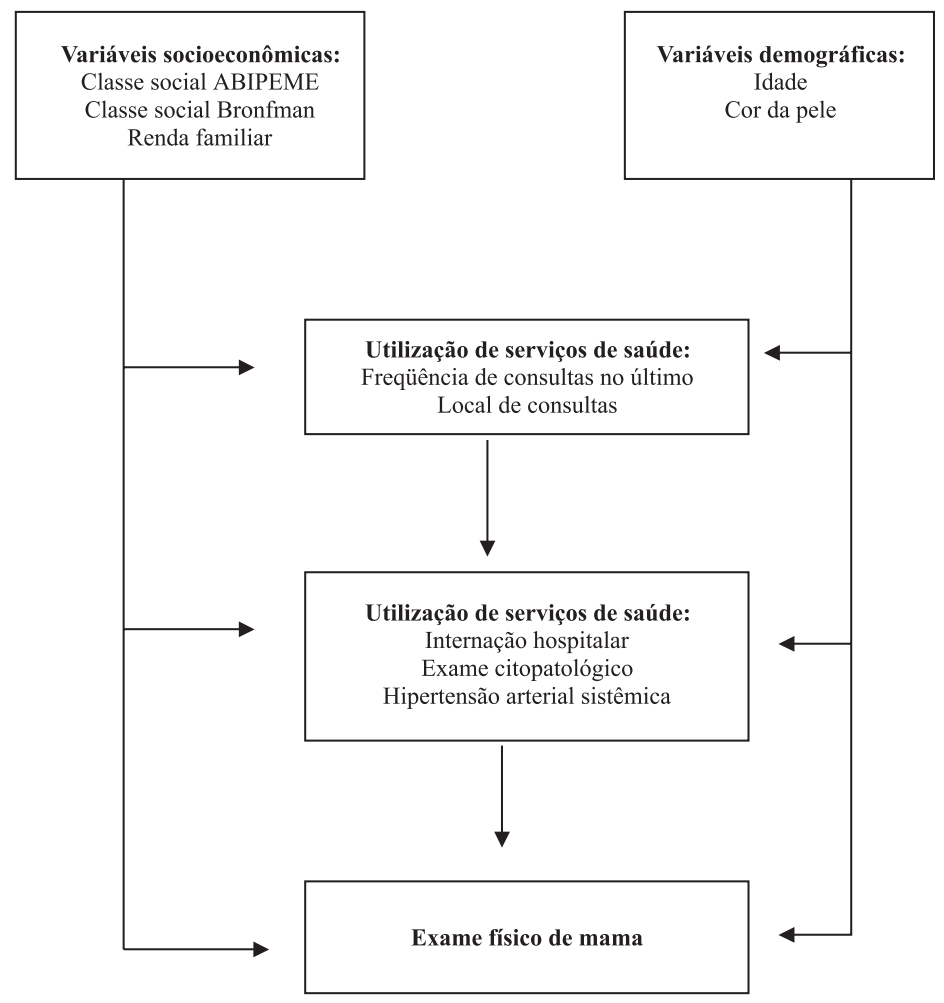

Figura 1. Modelo de análise hierarquizada.

Figure 1. Hierarchical analysis model.

tivas apenas entre mulheres que auferiam menos de um salário e mais do que dez salários (Tabela 1).

À medida que diminuia a escolaridade das mulheres, foi-se encontrando uma maior probabilidade de que não tivessem as mamas examinadas (Tabela 1 ).

Deve-se destacar que nas variáveis contínuas, classe social da ABIPEME, renda familiar e escolaridade, os testes de linearidade foram estatisticamente significativos.

Mulheres cuja cor da pele foi classificada como não branca foram menos freqüentemente examinadas do que as brancas (Tabela 1).

As mulheres com idade até 35 anos foram menos examinadas, porém não foram observadas diferenças estatisticamente significativas entre os dois grupos etários (Tabela 1).

A menor proporção de exame de mama realizado foi associada a menor utilização de serviços de saúde, caracterizada pelo menor número de consultas no último ano e exame citopatológico atrasado. Por outro lado, as mulheres que estiveram hospitalizadas no último ano foram mais examinadas do que aquelas que não foram internadas (Tabela 1).

Não foram encontradas diferenças estatisticamente significativas em relação ao local de consulta médica e se as mulheres apresentavam hipertensão arterial sistêmica (Tabela 1).

A Tabela 2 apresenta as características associadas à realização do exame de mamas ajustadas para fatores de confusão, através de regressão logística. Foi destacado que a realização de uma a duas consultas está associada, de maneira significativa e independentemente de características socioeconômicas e demográficas, a uma maior chance de não ser realizado o exame de mama. Foi verificado que as mulheres que estavam com exame citopatológico atrasado, mesmo após ajuste para as variáveis socioeconômicas 
Tabela 1, Distribuição da amostra e características associadas a ausência de exame médico de mama, Pelotas, RS, 1999-2000. Table 1, Distribution of the sample and characteristics associated with breast exam not being performed by doctors, Pelotas, $R S$, 1999-2000.

\begin{tabular}{|c|c|c|c|c|c|}
\hline Variável & $\begin{array}{c}\mathrm{N} \\
\text { médico de } \\
\text { mama n (\%) }\end{array}$ & $\begin{array}{l}\text { Sem exame } \\
\text { prevalências }\end{array}$ & Razão de & IC de $95 \%$ & valor $p$ \\
\hline \multicolumn{6}{|l|}{ Classe social (ABIPEME)* } \\
\hline Classe A & 60 & $14(23,3)$ & 1,0 & & \\
\hline Classe B & 281 & $109(38,8)$ & 1,66 & $1,03-2,69$ & \\
\hline Classe C & 403 & $188(46,7)$ & 2,00 & $1,25-3,20$ & \\
\hline Classe D & 319 & $198(62,1)$ & 2,66 & $1,67-4,24$ & \\
\hline Classe E & 49 & $34(69,4)$ & 2,97 & $1,81-4,88$ & $<0,001$ \\
\hline \multicolumn{6}{|l|}{ Classe social (Bronfman) } \\
\hline $\begin{array}{l}\text { Burguesia e nova e } \\
\text { pequena burguesia }\end{array}$ & 149 & $48(32,2)$ & 1,0 & $1,20-2,05$ & \\
\hline Pequena burguesia tradicional & 222 & $112(50,5)$ & 1,57 & $1,12-1,86$ & \\
\hline Proletariado não típico & 460 & $214(46,5)$ & 1,44 & $1,38-2,38$ & \\
\hline Proletariado típico & 132 & $77(58,3)$ & 1,81 & $1,47-2,51$ & \\
\hline Subproletariado & 142 & $88(62,0)$ & 1,92 & & $<0,001$ \\
\hline \multicolumn{6}{|l|}{ Renda familiar per capita em SM* } \\
\hline$>10,00$ & 56 & $20(35,7)$ & 1,0 & $0,54-1,41$ & \\
\hline $6,01-10,00$ & 80 & $25(31,1)$ & 0,88 & $0,66-1,49$ & \\
\hline $3,01-6,00$ & 166 & $59(35,5)$ & 1,00 & $0,98-2,03$ & \\
\hline $1,01-3,00$ & 456 & $230(50,4)$ & 1,41 & $1,14-2,36$ & \\
\hline$<1,01$ & 356 & $209(58,7)$ & 1,64 & & $<0,001$ \\
\hline \multicolumn{6}{|l|}{ Escolaridade (anos)* } \\
\hline$\geq 15$ & 119 & $32(26,9)$ & 1,0 & $1,10-2,12$ & \\
\hline $11-14$ & 266 & $109(41,0)$ & 1,52 & $1,45-2,76$ & \\
\hline $8-10$ & 201 & $108(53,7)$ & 2,00 & $1,46-2,76$ & \\
\hline $5-7$ & 234 & $126(53,8)$ & 2,00 & $1,57-2,93$ & \\
\hline $0-4$ & 302 & $174(57,6)$ & 2,14 & & $<0,001$ \\
\hline \multicolumn{6}{|l|}{ Cor da pele } \\
\hline Branca & 932 & $441(47,3)$ & 1,0 & $1,04-1,38$ & \\
\hline Não branca & 190 & $108(56,8)$ & 1,20 & & $<0,05$ \\
\hline \multicolumn{6}{|l|}{ Idade } \\
\hline Menos de 35 anos & 353 & $181(51,3)$ & 1,0 & & \\
\hline De 35 a 69 anos & 769 & $368(47,9)$ & 1,07 & $0,94-1,21$ & 0,29 \\
\hline \multicolumn{6}{|l|}{ Local de consulta no último mês } \\
\hline Sistema privado & 88 & $32(36,4)$ & 1,0 & & \\
\hline Seguros privados & 110 & $30(27,3)$ & 0,75 & $0,50-1,13$ & \\
\hline Serviços contratados & 46 & $16(34,8)$ & 0,96 & $0,59-1,55$ & \\
\hline Sistema público & 104 & $40(38,5)$ & 1,06 & $0,73-1,53$ & 0,34 \\
\hline \multicolumn{6}{|l|}{ Internação hospitalar no último ano } \\
\hline Não & 977 & $493(50,5)$ & 1,0 & & \\
\hline Sim & 145 & $56(38,6)$ & 0,77 & $0,62-0,95$ & $<0,01$ \\
\hline \multicolumn{6}{|l|}{ Exame citopatológico atrasado } \\
\hline Não & 810 & $297(36,7)$ & 1,0 & & \\
\hline Sim & 312 & $252(80,8)$ & 2,20 & $1,8-2,45$ & $<0,001$ \\
\hline \multicolumn{6}{|l|}{ Consultas médicas no último ano } \\
\hline Três ou mais & 546 & $205(37,5)$ & 1,0 & & \\
\hline De uma a duas & 355 & $159(44,8)$ & 1,19 & $1,02-1,40$ & $<0,001$ \\
\hline \multicolumn{6}{|l|}{ Hipertensão Arterial Sistêmica } \\
\hline Não & 837 & $415(49,6)$ & 1,0 & & \\
\hline Sim & 281 & $131(46,6)$ & 1,04 & $0,88-1,21$ & 0,28 \\
\hline
\end{tabular}

*Teste de tendência linear $<0,001 /{ }^{*}$ Test of linear trend $<0.001$ 
(classe social da ABIPEME e renda familiar), demográficas (cor da pele) e freqüência de consultas médicas no último ano, apresentaram um risco significativamente maior de não terem as mamas examinadas. Contudo, o efeito protetor da hospitalização no último ano sobre a realização do exame de mama desapareceu após o controle para fatores de confusão (Tabela 2).

A análise multivariada também foi realizada utilizando-se a classificação de classe social de Bronfman, em vez da ABIPEME, como variável socioeconômica hierarquicamente mais importante. Os resultados foram semelhantes e confirmaram a associação entre a freqüência de consultas médicas no último ano, o exame citopatológico e a realização do exame de mama.
Foram comparados nos dois períodos os percentuais de mulheres não examinadas, segundo a classificação da ABIPEME, através de seus respectivos intervalos de confiança. Em ambos os períodos, os dados revelaram diferenças entre as classes. Em 1992, as mulheres inseridas nas classes A e B foram mais examinadas do que aquelas pertencentes às categorias D e E. Em 2000, as mulheres da classe mais elevada foram mais examinadas do que aquelas classificadas nas outras classes. Contudo, os percentuais de não realização do exame em 1992 foram sempre inferiores aos do ano 2000.

\section{Discussão}

Os vieses inerentes aos estudos epide-

Tabela 2. Análise por regressão logística da não realização de exame médico de mama.

Pelotas, RS, 1999-2000

Table 2. Logistic regression analyses in the sample without breast exam by doctor. Pelotas, RS, 1999-

2000

\begin{tabular}{|c|c|c|c|}
\hline Variável & Odds ratio & Intervalo de confiança & P-Valor \\
\hline \multicolumn{4}{|c|}{ Classe social (ABIPEME) } \\
\hline Classe A & 1,0 & & \\
\hline Classe B & 2,08 & $1,09-3,96$ & \\
\hline Classe C & 2,87 & $1,53-5,39$ & \\
\hline Classe D & 5,37 & $2,83-10,19$ & \\
\hline Classe $\mathrm{E}$ & 7,44 & $3,17-17,47$ & $<0,001$ \\
\hline \multicolumn{4}{|c|}{ Renda familiar per capita em SM } \\
\hline$>10,00 \mathrm{SM}$ & 1,0 & & \\
\hline 6,01 a $10,00 \mathrm{SM}$ & 0,81 & $0,39-1,68$ & \\
\hline 3,01 a 6,00 SM & 0,99 & $0,52-1,86$ & \\
\hline 1,01 a $3,00 \mathrm{SM}$ & 1,83 & $1,02-3,26$ & \\
\hline$<1,01 \mathrm{SM}$ & 2,56 & $1,42-4,59$ & $<0,001$ \\
\hline \multicolumn{4}{|l|}{ Cor da pele } \\
\hline Branca & 1,0 & & \\
\hline Não branca & 1,46 & $1,07-2,00$ & 0,05 \\
\hline \multicolumn{4}{|c|}{ Consultas médicas no último ano* } \\
\hline Três ou mais & 1,0 & & \\
\hline De uma a duas & 1,32 & $1,00-1,76$ & 0,04 \\
\hline \multicolumn{4}{|c|}{ Exame citopatológico atrasado +} \\
\hline Não & 1,0 & & \\
\hline Sim & 5,22 & $3,62-7,52$ & $<0,001$ \\
\hline \multicolumn{4}{|c|}{ Internação hospitalar no último ano + } \\
\hline Sim & 0,85 & $0,56-1,31$ & 0,39 \\
\hline
\end{tabular}


miológicos de base populacional foram aparentemente controlados neste estudo ${ }^{19}$. A estrutura da amostra por faixa etária foi semelhante àquela projetada pelo IBGE para a zona urbana da cidade de Pelotas, o que confirmou a representatividade do estudo e demonstrou que não houve viés de seleção. Assim como foram controlados os possíveis fatores de confusão na regressão logística.

A análise desse estudo partiu do princípio de que o exame físico de mamas deve fazer parte de toda consulta médica, qualquer que seja a especialidade, atentando-se para os grupos de risco.

Os resultados deste estudo indicaram baixa prevalência de realização do exame físico de mama no ano 2000. Destacou-se, portanto, uma diminuição de $20 \%$ na realização do exame de mama por médicos nos últimos sete anos. Estes resultados sugeriram a ausência de impacto da campanha denominada Dia M, realizada pela Secretaria de Saúde e do Meio Ambiente há cerca de cinco anos, na qual as mulheres são submetidas ao exame de mama e recebem instruções sobre como realizar o auto-exame ${ }^{7}$. Além disso, a diminuição na cobertura indica a falta de integralidade na atenção ambulatorial na cidade de Pelotas. Numa cidade com extensa rede de atendimento ambulatorial, onde se sabe que as mulheres consultam em média 3,9 vezes por ano, a cobertura de realização do exame poderia ser mais elevada ${ }^{12}$.

Em 1992, foi mostrado que as mulheres que consultavam na rede privada eram mais freqüentemente examinadas do que aquelas que procuravam o sistema público ${ }^{11}$. No presente estudo, não foram encontradas di- ferenças de realização do exame de mamas associadas ao tipo de serviço, público ou privado. Considerando-se a diminuição da cobertura, o achado sugere a desqualificação da rede ambulatorial.

Outra deficiência da assistência médica ambulatorial da cidade de Pelotas detectada neste estudo refere-se à falta de conhecimento e/ou aplicação do conceito de risco epidemiológico fundamentando os procedimentos médicos. As mulheres mais idosas sabidamente apresentam maior risco de desenvolver câncer de mama, e portanto deveriam ser examinadas mais vezes pelos médicos ${ }^{20}$. Contudo, não foram encontradas diferenças estatisticamente significativas na freqüência de realização do exame entre as mulheres mais jovens e aquelas com 35 anos ou mais. Por outro lado, as mulheres com 35 anos ou mais foram as que mais freqüentemente realizaram o auto-exame de mama, ou seja, este grupo parece perceber a necessidade da prática preventiva.

Foi constatado positivamente que o aumento de freqüência aos serviços de saúde aumentou a cobertura do exame físico de mama.

As mulheres que tinham exame citopatológico atualizado foram mais examinadas do que aquelas com o procedimento realizado há mais de três anos. Por outro lado, um percentual elevado (37\%) de mulheres com exame citopatológico atualizado não teve as mamas examinadas, sugerindo, novamente, a falta de integralidade dos procedimentos preventivos.

Tentou-se relacionar a realização do exame com internações hospitalares no último ano e presença de hipertensão arterial

Tabela 3. Percentual das mulheres sem exame médico de mamas e seus respectivos intervalos de confiança. Pelotas, 1992 e 2000.

Table 3. Percentage of women without medical breast exam and respective confidence intervals. Pelotas, 1992 and 2000.

\begin{tabular}{lcc}
\hline VARIÁVEL & $1992\left(\mathrm{IC}_{95 \%}\right)$ & $2000\left(\mathrm{IC} \mathrm{C}_{95 \%}\right)$ \\
\hline CLASSE A e B & $8,1(1,9-14,3)$ & $36,0(31,0-41,2)$ \\
CLASSE C & $15,5(10,2-20,8)$ & $46,7(41,8-51,6)$ \\
CLASSE D & $19,3(14,5-24,1)$ & $62,1(56,8-67,4)$ \\
CLASSE E & $31,8(25,4-38,2)$ & $69,4(56,5-82,3)$ \\
\hline
\end{tabular}


sistêmica, pois são duas condições associadas a uma maior freqüência de consultas médicas e, por conseqüência, devem indicar cuidados mais freqüentes ${ }^{12}$. Entretanto, $38 \%$ das mulheres foram hospitalizadas no último ano e não tiveram suas mamas examinadas, evidenciando também a deficiência no nível de maior complexidade.

Pode-se criticar a prática do exame físico de mama alegando-se que a mamografia é um método mais acurado e que deveria ser realizado anualmente nas mulheres entre 40 e 69 anos $^{5}$. Segundo dados do IBGE, a população feminina em Pelotas, entre 40 e 69 anos, totaliza 46.842 pessoas. O valor pago pelo Sistema Único de Saúde para cada mamografia é de R\$ 30,12. Pela imprensa local, sabe-se que a estimativa orçamentária para o exercício do ano 2000, com recursos próprios, atinge a quantia de $\mathrm{R} \$ 14.979 .000,00$. Desta forma, o custo total de se fazer mamografia anual nas mulheres entre 40 e 69 anos atingiria o valor de $\mathrm{R} \$ 1.410 .881,00$, consumindo em apenas um programa aproximadamente $10 \%$ do orçamento da saúde para o município. Portanto, numa cidade pobre com inúmeras necessidades, deve-se levar em conta os custos dos procedimentos e torna-se fundamentalmente necessária a valorização de cuidados preventivos representados pelo exame de mama.

\section{Conclusões}

Eliminar as diferenças no acesso à atenção médica, no recebimento de cuidados médicos e, conseqüentemente, nos padrões de morbi-mortalidade entre pessoas ricas e pobres tem sido colocado entre as prioridades para os sistemas de saúde ${ }^{21}$. Neste estudo, foi investigado o efeito das características socioeconômicas na realização de cuidados primários. Foram escolhidas algumas variáveis que expressam condições sociais e de vida, nas quais verificou-se que quanto mais baixa a inserção de classe e a escolaridade, ou pior o nível de renda, menor a proporção de mulheres examinadas. Este achado evidencia uma indesejável iniqüidade no sistema local de saúde, remetendo a diversas considerações políticas sobre a falta de justiça social e compromisso com a população assistida ${ }^{22}$. Corroborando este achado, foi comprovado que as mulheres brancas são mais freqüentemente examinadas do que as não brancas, o que, de certa forma, enfatiza as diferenças socioeconômicas, previamente discutidas.

\section{Referências}

1. Ministério da Saúde. Secretaria Nacional de Assistência à Saúde. Instituto Nacional do Câncer. Estimativa de incidência e mortalidade por câncer no Brasil. Rio de Janeiro: Instituto Nacional do Câncer; 1999.

2. Secretaria da Saúde e do Meio Ambiente. Núcleo de Informação em Saúde. Estatísticas de Saúde: mortalidade 1995. Porto Alegre: Secretaria da Saúde e do Meio Ambiente; 1996.

3. Secretaria da Saúde. Coordenadoria de Informações em Saúde. Estatísticas de Saúde: mortalidade 2000. Porto Alegre: Secretaria da Saúde; 2001.

4. Harris JR, Lippman ME, Veronesi U, Willet W. Breast Cancer (First of Three Parts). N Engl J Med 1992; 327(5): 319-28.
5. Conelly MT, Inui TS. Principles of Disease Prevention. In: Fauci AS, Braunwald E, Isselbacher KJ, Wilson JD, Kasper DL, Hauser SL et al. (eds). Harrison's Principles of Internal Medicine. New York: The McGraw-Hill Companies; 1998. p.46-8.

6. McPhee SJ, Schroeder AS. General Aproach to the Patient; Health Maintenance \& Disease Prevention; \& Common Symptoms. In: Tierney Jr LM, McPhee SJ, Papadakis MA (eds). Current Medical Diagnosis \& Treatment 1999. Stamford: Appleton \& Lange; 1999. p.1-32.

7. Secretaria da Saúde e do Meio Ambiente. Seção de Saúde da Mulher. Programa de Controle do Câncer de Mamas. Normas Técnicas e Operacionais. Porto Alegre: Secretaria da Saúde e do Meio Ambiente; 1997. 
8. Majeed FA, Voss S. Performance indicators for general practice. BMJ 1995; 311:209-10.

9. McColl A, Roderick P, Gabbay J, Smith H, Moore M. Performance indicators for primary care groups: an evidence based approach. BMJ 1998; 317: 1354-60.

10. Walt G. Health policy: an introduction to process and power. London: Zed Books; 1998.

11. Dias da Costa JS, Piccini RX, Moreira MR. Avaliação da prática do auto-exame e exame físico de mamas na cidade de Pelotas-RS. Rev Bras Ginec Obstet 1995; 17(6): 621-32.

12. Dias da Costa JS, Facchini LA. Utilização de serviços ambulatoriais em Pelotas: onde a população consulta e com que freqüência. Rev Saúde Pública 1997; 31(4): 360-9.

13. Dias da Costa JS, Roman VR, Luz RM, Manzolli PP, Correa P, Recuero LF. Auditoria Médica: avaliação de alguns procedimentos inseridos no Programa de Atenção à Saúde da Mulher no Posto de Saúde da Vila Municipal, Pelotas, RS. Cad Saúde Pública 1998; 14(1): 43-9.

14. Bronfman M, Lombardi C, Facchini LA, Victora CG, Barros FC, Béria JU et al. Operacionalização do conceito de classe social em estudos epidemiológicos. Rev Saúde Pública 1988; 22: 253-65.
15. Rutter M. Pesquisa de Mercado. São Paulo: Ed. Ática; 1988.

16. Consenso Brasileiro de Hipertensão, 3. Campos do Jordão, S.P.: Sociedade Brasileira de Hipertensão, 12 15 Fev. 1998.

17. Altman DG. Practical Statistics for Medical Research. London: Chapman \& Hall; 1997.

18. Victora CG, Huttly SR, Fuchs SC, Olinto MTA. The role of conceptual frameworks in epidemiological analysis: a hierarchical approach. Int J Epidemiol 1997; 26: 22447 .

19. Albhom A, Norell S. Introduction to Modern Epidemiology. Chestnut Hill: Epidemiology Resources Inc.; 1990.

20. Boring CC, Squires TS, Tong T, Montgomery S. Cancer Statistics, 1994. CA Cancer J. Clin. 1994; 44(1): 7-26.

21. Alleyne GAO. Equity and health. Pan Am J Public Health 2000; 7(1): 1-7.

22. Berlinguer G. Population, ethics and equity. Cad Saúde Pública 1999; 15(Supl.1): 111-22.

Recebido em: 07/12/01 $1^{\text {a }}$ aprovação em: 08/04/02 Versão final em: 24/06/02 